\title{
The psychological status of frontline health workers confronting COVID-19 after local cases eradication in Zhuhai, Southern China
}

\author{
Yingjian Liang ${ }^{1} \cdot$ Meizhu Chen ${ }^{1} \cdot$ Xiaobin Zheng $^{1} \cdot$ Changli Tu $^{1} \cdot$ Cuiyan Tan ${ }^{1} \cdot$ Jing Liu ${ }^{1}$
}

Received: 4 August 2020 / Accepted: 14 October 2020 / Published online: 1 November 2020

○) Springer-Verlag GmbH Germany, part of Springer Nature 2020

Our previous work has showed that several medical staff were experiencing clinically significant depressive and anxious symptoms during the outbreak of coronavirus disease 2019 (COVID-19) and Chen and his colleagues outlined some essentially psychological interventions $[1,2]$. However, scanty data about the psychological status of them after return to daily medical activities as local cases eradicating, which are of interest.

The Fifth Affiliated Hospital of Sun Yat-sen University, a territory hospital of Zhuhai in a southern coast of China (>1500 beds), cleared its local COVID-19 confirmed cases on 10 March 2020. Mental health of the medical staff with or without COVID-19 contacting history was assessed by Zung's self-rating depression scale (SDS) and Zung's selfrating anxiety scale (SAS) from 11 to 16 March 2020. This study was approved by the Research Ethics Committee of the Fifth Affiliated Hospital of Sun Yat-sen University (K17-1).

In total, we collected data from 51 nurses and 19 doctors, including 44 frontline medical workers and 26 control subjects (medical staff never served for COVID-19 cases). Similar to prior data, there is insignificant difference between the SDS and SAS raw scores of younger and the older staff $\left(\mathrm{SDS}, t\right.$ test, mean $_{\leq 30}=29.35$, $\mathrm{man}_{>30}=30.42, P=0.56$; SAS t-test, mean $_{\leq 30}=27.54$, mean $_{>30}=28.13, P=0.72$ ) as well as the COVID-19 contacting and non-contacting (SDS, $t$ test, mean $_{\text {frontline }}=29.24$, mean $_{\text {control }}=30.56, P=0.47$; SAS $t$ test, mean $_{\text {frontline }}=27.16$, mean $_{\text {control }}=28.80, P=0.30$ ). Moreover, several health workers were in mild-to-moderate depressive or anxious state based on established threshold. As illustrated, 27 participants were tracked in previous study (13 frontline staff). Regarding control group, nearly, half (8/14 in SDS and 7/14 in SAS) of their scores descend and one is still in mild depression. Regarding the frontline, most of their SDS scores (9/13) increased, whereas most SAS scores conversely decreased (11/13). One of the frontline staff is still in mild anxiety (Fig. 1).

Several reports about mental problems in Chinese health care workers are available, since they were under heavy physical and psychological pressure during the coronavirus pandemic $[3,4]$. Awareness of the level of depression as well as anxiety of COVID-19 frontline medical staff can effectively help psychological counselors provide adjusted support accordingly. In this present study, we found that depressive and anxious symptoms still persisted after our local cases eradication, which indicated that long-term further measures of psychological intervention, such as family support, peer support meetings or group psychotherapy, are desirable [5]. However, our analysis made it different to conclude whether the mental burden is temporal or long-lasting. Perhaps psychologic health surveillance of about frontline medical staff are warranted.

Jing Liu

liujing25@mail.sysu.edu.cn

1 Department of Pulmonary and Critical Care Medicine (PCCM), Fifth Affiliated Hospital of Sun Yat-Sen University, 52 East Meihua Rd, Zhuhai City 519000, China 

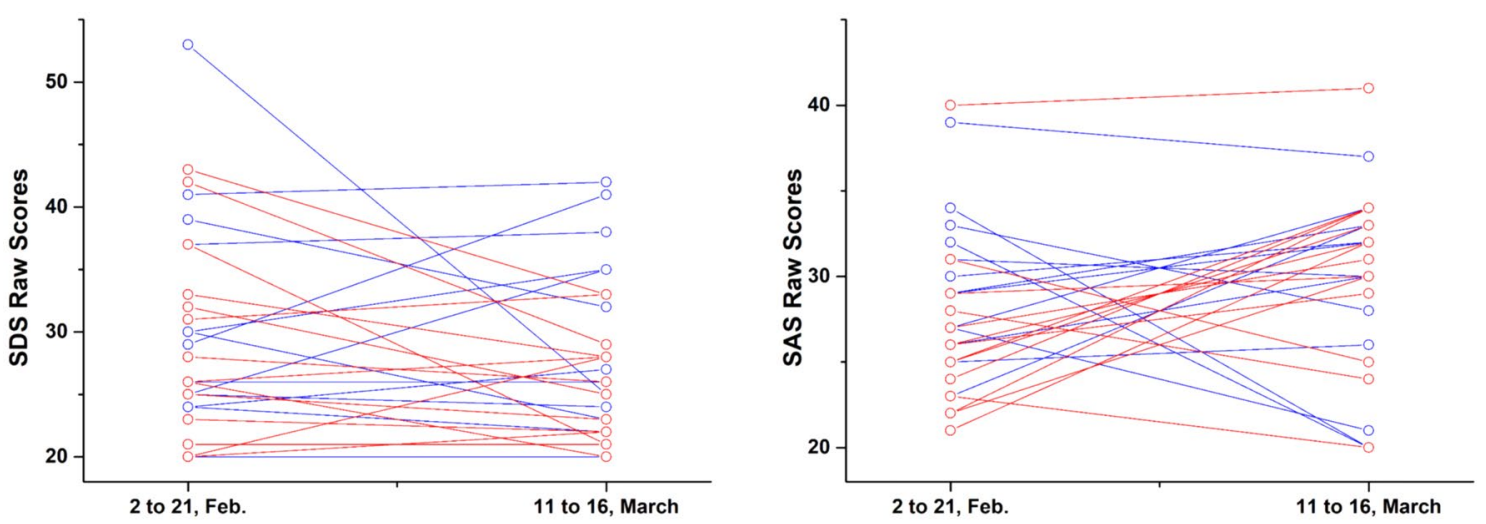

Fig. 1 Raw scores of SDS and SAS among frontline healthcare workers as well as control subject during COVID-19 outbreak and after Zhuhai city local cases clearing. Blue represents frontline healthcare workers. Red represents control subject

Acknowledgements This work was supported by the Emergency project of clinical research on the prevention and treatment of COVID19 with traditional Chinese medicine from Guangdong provincial bureau of traditional Chinese medicine, China (grant numbers 2020ZYYJ16), Guangdong Natural Science Foundation (grant number (2020A151501153), the Fundamental Research Funds for the Central Universities (grant numbers 19ykpy51), Open project of Key Laboratory of Tropical Disease Control (Sun Yat-sen University), Ministry of Education (grant number 2020kfkt04/07) and Zhuhai science and technology innovation Bureau (grant number ZH22036302200021PWC, 2019.02-2019.12).

\section{Compliance with ethical standards}

Conflicts of interest The authors report no conflicts of interest in this work. All authors have read and approved the manuscript.

\section{References}

1. Chen Q, Liang M, Li Y, Guo J, Fei D, Wang L, He L, Sheng C, Cai Y, Li X, Wang J, Zhang Z (2020) Mental health care for medical staff in China during the COVID-19 outbreak. Lancet Psychiatry 7:e15-e16

2. Liang Y, Chen M, Zheng X, Liu J (2020) Screening for Chinese medical staff mental health by SDS and SAS during the outbreak of COVID-19. J Psychosom Res 133:110102

3. Kang L, Li Y, Hu S, Chen M, Yang C, Yang BX, Wang Y, Hu J, Lai J, Ma X, Chen J, Guan L, Wang G, Ma H, Liu Z (2020) The mental health of medical workers in Wuhan, China dealing with the 2019 novel coronavirus. Lancet Psychiatry 7:e14

4. Spoorthy MS, Pratapa SK, Mahant S (2020) Mental health problems faced by healthcare workers due to the COVID-19 pandemic - A review. Asian J Psychiatr 51:102119

5. Moreno C, Wykes T, Galderisi S, Nordentoft M, Crossley N, Jones N, Cannon M, Correll CU, Byrne L, Carr S, Chen E, Gorwood P, Johnson S, Kärkkäinen H, Krystal JH, Lee J, Lieberman J, LópezJaramillo C, Männikkö M, Phillips MR, Uchida H, Vieta E, Vita A, Arango C (2020) How mental health care should change as a consequence of the COVID-19 pandemic. Lancet Psychiatry $7: 813-824$ 